\title{
Chronische Diarrhoe beim Hund
}

\author{
Dorothee Dahlem, Iwan Burgener
}

Die chronische Diarrhoe kann bei Hunden durch zahlreiche Erkrankungen bedingt sein. Die diagnostische Aufarbeitung dieser Patienten ist häufig langwierig und kostenintensiv, und die Prognose für chronische Enteropathien im Allgemeinen ist schwierig vorauszusehen. Da differenzialdiagnostisch aber auch gut kontrollierbare Erkrankungen in Betracht kommen, sollten die Besitzer immer zu einer Diagnostik ermutigt werden.

\section{Definition}

Der Darm-Trakt ist nicht nur Ort der Verdauung, sondern auch ein immunologisches Organ. Aufgrund dieser vielfältigen Aufgaben sind kleinere Veränderungen der Kotkonsistenz normal [12,17]. Eine signifikante Erhöhung der Kotabsatzfrequenz oder des Flüssigkeitsanteils der Fäzes bzw. auch Kombinationen dieser Veränderungen werden als Diarrhoe definiert [12]. Diarrhoe wird durch eine verminderte intestinale $\mathrm{Ab}$ sorption oder eine gesteigerte intestinale Sekretion von Wasser hervorgerufen und ist somit immer Anzeichen einer primären oder sekundären intestinalen $\mathrm{Er}$ krankung. Das Fehlen von Durchfall schließt eine Erkrankung des MagenDarm-Traktes allerdings nicht aus.

Als chronisch gilt Diarrhoe, wenn sie seit mindestens 14 Tagen besteht. Zudem zählt auch das episodische Auftreten von Diarrhoe zu diesem Begriff [34].

\section{Klassifizierung}

Neben akuten und chronischen Durchfallerkrankungen unterscheidet man zwischen Dünn- und Dickdarmdiarrhoe, wobei häufig auch Mischformen vorkommen [34]. Wichtige Kriterien zur Unterscheidung zwischen Dünn- und Dickdarmdiarrhoe sind:
- Vomitus

- Gewichtsverlust

- Hämatochezie

- schleimige Kotbeimengungen

Der Dünndarm fungiert vor allem als Resorptionsort für Nährstoffe, weshalb Gewichtsverlust bei Patienten mit chronischem Dünndarmdurchfall häufig zu beobachten ist. Vomitus wird ebenfalls häufiger in Zusammenhang mit Dünndarmdurchfall beobachtet. Hämatochezie und Beimengungen von schleimigem Sekret treten vor allem bei Dickdarmdurchfall auf. Sofern das Rektum mitbetroffen ist, kann auch Tenesmus vorkommen ( Tab. 1) [1, 12].

Bei chronischem Dünndarmdurchfall muss weiterhin differenziert werden, ob eine Maldigestion oder eine Malabsorpeiner Maldigestion beim Hund zählt die exokrine Pankreasinsuffizienz. Aufgrund des Fehlens von Verdauungsenzymen ist eine Aufspaltung der aufgenommenen Nährstoffe bei dieser Erkrankung nicht tion vorliegt. Zu den häufigsten Ursachen

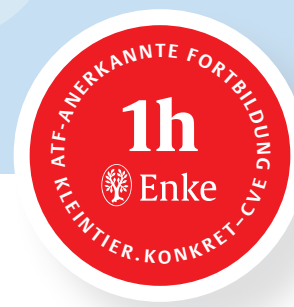

adäquat möglich. Bei malabsorptiven Erkrankungen, bei denen Nährstoffe aufgespalten, aber nicht adäquat resorbiert werden können, unterscheidet man zwischen Proteinverlustenteropathien (PVE) und Enteropathien ohne Proteinverlust [34].

\section{Ätiologie}

Die Ursachen der chronischen Diarrhoe beim Hund lassen sich grundlegend in extraintestinale und intestinale Ursachen unterscheiden ( $\vee$ Tab.2). Extraintestinale Erkrankungen führen über mukosaschädigende Stoffwechselprodukte zu Durchfall. Ein Beispiel hierfür ist die urämische Gastroenteritis bei Patienten mit Azotämie. $\mathrm{Zu}$ den wichtigsten extraintestinalen Ursachen chronischer Diarrhoe zählen exokrine Pankreasinsuffizienz, chronisch-rezidivierende Pankreatitis, hepatobiliäre Er-

Tab. 1 Unterscheidung zwischen Dünn- und Dickdarmdiarrhoe.

\begin{tabular}{|c|c|c|}
\hline & Dünndarmdiarrhoe & Dickdarmdiarrhoe \\
\hline Gewichtsverlust & häufig & selten \\
\hline $\begin{array}{l}\text { Defäkations- } \\
\text { frequenz }\end{array}$ & $\begin{array}{l}\text { normal bis leicht erhöht } \\
\text { (ca. } 2-4 \times \text { pro Tag) }\end{array}$ & $\begin{array}{l}\text { stark erhöht } \\
\text { (ca. } 4-12 \times \text { pro Tag) }\end{array}$ \\
\hline Menge pro Absatz & normal bis erhöht & meist reduziert \\
\hline Vomitus & häufig & möglich (ca. 35\% bei Kolitis) \\
\hline Appetit & meist normal bis gesteigert & normal bis vermindert \\
\hline $\begin{array}{l}\text { Hämatochezie } \\
\text { (frisches Blut) }\end{array}$ & selten & häufig \\
\hline Meläna (Teerstuhl) & möglich & abwesend \\
\hline Schleim & sehr selten & häufig \\
\hline Futter & teils unverdaut & immer verdaut \\
\hline $\begin{array}{l}\text { mögliche Begleit- } \\
\text { erscheinungen }\end{array}$ & $\begin{array}{l}\text { Borborygmus, Flatulenz, } \\
\text { Steatorrhoe (Fettstuhl) }\end{array}$ & $\begin{array}{l}\text { Tenesmus, Dyschezie, } \\
\text { Kotinkontinenz }\end{array}$ \\
\hline
\end{tabular}


krankungen, Nierenerkrankungen und Hypoadrenokortizismus [12].

Endoparasitosen, fehlerhafte Fütterung, abrupter Wechsel der Diät sowie idiopathische intestinale Entzündungen (IBD) und intestinale Neoplasien sind häufige Ursachen für intestinale Diarrhoe [34]. $\mathrm{Zu}$ den intestinalen Ursachen zählen zudem bakterielle Erkrankungen, wobei die klinische Bedeutung primärer bakterieller Erkrankungen des Magen-DarmTraktes sehr kritisch zu diskutieren ist. Entscheidend ist dabei in erster Linie die Zusammensetzung der intestinalen Flora und nicht die absolute Zahl bakterieller Erreger [11, 29].

Eine Ausnahme sind spezifische pathogene Erreger, die teilweise auch eine zoonotische Bedeutung haben (z.B. Salmonella spp. und Campylobacter spp. [17]). Die durch diese Erreger ausgelösten Erkrankungen haben aber gewöhnlich einen akuten Verlauf.

\section{.konkret}

Eine intestinale Dysbakterie ist bei Durchfallerkrankungen immer nachweisbar, sie stellt i.d. R. aber nicht die Ursache, sondern die Folge einer anderweitigen Darmerkrankung dar.

\section{Klinische Symptome}

Häufig zeigen Hunde mit chronischer Diarrhoe keine weiteren klinischen Symptome. Gewichtsverlust, fehlende Gewichtzunahme, Vomitus und Inappetenz können bei Hunden mit Dünndarmdurchfall auftreten. Auch abdominale Dolenz, rezidivierende Tympanie, Borborygmus, Flatulenz und Salivation sind Hinweise auf Dünndarmerkrankungen. Seltener treten eine kompensatorische Polydipsie, Polyphagie und Koprophagie auf [12]. Chronische Durchfallpatienten, die mit Dehydratation, Apathie und Abmagerung vorgestellt werden, befinden sich oft in einem späten Stadium der Erkrankung oder weisen eine schwerwiegende Grunderkrankung auf (Neoplasie, PVE).

Hunde mit chronischem Dickdarmdurchfall werden häufig aufgrund von Tenesmus oder erhöhter Kotabsatzfrequenz vorgestellt. Meist zeigen Tiere mit

Tab. 2 Differenzialdiagnosen der Diarrhoe beim Hund.

\begin{tabular}{|c|c|}
\hline intestinale Ursachen & extraintestinale Ursachen \\
\hline Parasiten & Nephropathien \\
\hline $\begin{array}{l}\text { Bakterien } \\
\text { - Campylobacter spp. } \\
\text { - Clostridium perfringens } \\
\text { - Salmonella spp. } \\
\text { - E. coli } \\
\text { - Helicobacter spp.? }\end{array}$ & Elektrolyt-|Säure-Basen-Störungen \\
\hline $\begin{array}{l}\text { Viren } \\
\text { - Parvoviren } \\
\text { - Coronaviren } \\
\text { Futtermittel-responsive Diarrhoe (FRD) }\end{array}$ & $\begin{array}{l}\text { systemische Infektionen } \\
\text { (Parvovirose) } \\
\text { - Staupe } \\
\text { - Leptospirose } \\
\text { - HCC }\end{array}$ \\
\hline Antibiotika-responsive Diarrhoe (ARD) & $\begin{array}{l}\text { Entzündungen } \\
\text { - Prostatitis } \\
\text { - Pyometra }\end{array}$ \\
\hline Inflammatory Bowel Disease (IBD) & $\begin{array}{l}\text { - Peritonitis } \\
\text { - Pyelonephritis }\end{array}$ \\
\hline \multicolumn{2}{|l|}{ Proteinverlustenteropathie (PVE) } \\
\hline histiozytäre ulzerative Kolitis (HUC) & $\begin{array}{l}\text { Endokrinopathien } \\
\text { - Hypoadrenokortizismus } \\
\text { - Diabetes mellitus, diabetische Ketoazidose } \\
\text { - Gastrinom }\end{array}$ \\
\hline \multicolumn{2}{|l|}{ Lymphangiektasie } \\
\hline $\begin{array}{l}\text { Neoplasien } \\
\text { - Lymphom } \\
\text { - Adenokarzinom } \\
\text { - Leiomyosarkom }\end{array}$ & $\begin{array}{l}\text { Medikamente } \\
\text { - Antibiotika } \\
\text { - NSAIDs } \\
\text { - Chemotherapeutika }\end{array}$ \\
\hline Fremdkörper, Ileus & $\begin{array}{l}\text { Toxine } \\
\text { - Ethylenglykol }\end{array}$ \\
\hline Motilitätsprobleme & neurologische Erkrankungen \\
\hline
\end{tabular}

isoliertem Dickdarmdurchfall aber ein ungestörtes Allgemeinbefinden. Bei der rektalen Untersuchung können Dolenz, eine verdickte Rektumschleimhaut und frischblutige Beimengungen festgestellt werden. Bei einer zugrunde liegenden Neoplasie sind möglicherweise rektale Umfangsvermehrungen palpierbar [1].

\section{Diagnostische Aufarbeitung}

Die diagnostische Aufarbeitung chronischer Durchfallpatienten ist häufig ein langwieriger Prozess und kann für den Patientenbesitzer sehr frustrierend sein, da die endgültige Diagnosestellung in erster Linie auf dem Ausschluss vieler Differenzialdiagnosen beruht. Die Notwendigkeit der einzelnen diagnostischen Schritte sollte vorher mit dem Besitzer intensiv besprochen werden, um Frustration und fehlender Compliance vorzubeugen. Das gute Allgemeinbefinden der Patienten mit chronischem Durchfall ermöglicht den für die Aufarbeitung erforderlichen Zeitrahmen von mehreren Wochen. Ausnahmen, in denen von dem klassischen diagnostischen Stufenplan ( $\triangleright$ Abb. 1) abgewichen werden sollte, sind in erster Linie besondere Befunde der klinischen Untersuchung (Palpationsbefund, schlechtes Allgemeinbefinden) und Hypalbuminämie.

Bei der diagnostischen Aufarbeitung chronischer Durchfallpatienten sollte zunächst den nicht-invasiven Maßnahmen der Vorzug gegeben werden. In der klinischen Untersuchung weisen viele Patienten mit chronischem Durchfall keine auffälligen Befunde auf. 


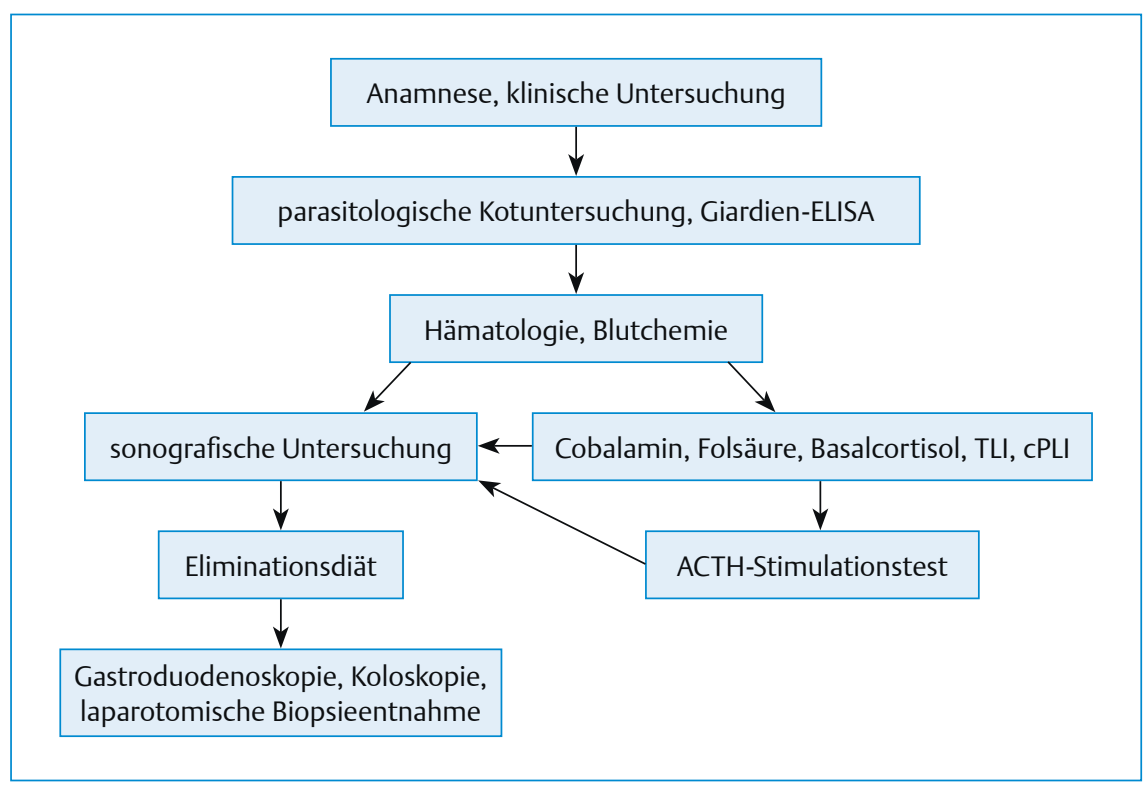

Abb. 1 Diagnostische Aufarbeitung der chronischen Diarrhoe beim Hund.

\section{Parasitologische Kotuntersuchung}

Gerade bei Jungtieren ist eine parasitologische Kotuntersuchung der 1. Schritt der diagnostischen Aufarbeitung. Bei gutem Allgemeinbefinden bietet es sich an, diese Untersuchung aus einer Sammelkotprobe (3 Tage) durchzuführen, um die Nachweiswahrscheinlichkeit intermittierend ausgeschiedener Entwicklungsstadien zu erhöhen. Die Kotuntersuchung sollte eine Sedimentation/Flotation und einen Giardien-ELISA beinhalten.

\section{konkret}

Auch bei fehlendem Parasitennachweis ist eine prophylaktische antiparasitäre Therapie anzuraten.

Gerade bei Giardieninfestation sind zudem besondere Hygienemaßnahmen erforderlich, und die Besitzer müssen über das zoonotische Potenzial aufgeklärt werden. Bei adulten Tieren führt eine Endoparasitose alleine selten zu chronischem Durchfall, kann aber eine zugrunde liegende anderweitige Erkrankung verschlimmern.

\section{Blutuntersuchungen}

Im Anschluss an die parasitologische Untersuchung sollten metabolische Ursachen abgeklärt werden.
Die hämatologische Untersuchung ist häufig unauffällig. Beim Fehlen eines Stressleukogramms trotz chronischer Erkrankung sollte ein Hypoadrenokortizismus in Betracht gezogen werden. Eine mikrozytäre hypochrome Anämie und eine Thrombozytose können Hinweise auf eine gastrointestinale Blutung inkl. Eisenverlust darstellen [24]. Für eine Eosinophilie kommen differenzialdiagnostisch in erster Linie eine Endoparasitose, eine allergische Erkrankung oder ein Hypoadrenokortizismus in Betracht $[8,20]$.

Mithilfe der blutchemischen Untersuchung können Hepatopathien und Nierenerkrankungen als Ursache der chronischen Diarrhoe abgeklärt werden. Die Bestimmung von Protein- und Elektrolytkonzentrationen ist zudem wichtig zum Nachweis möglicher Folgeerscheinungen der chronischen Diarrhoe. Eine Panhypoproteinämie ist hinweisend auf eine Proteinverlustenteropathie (PVE), während eine Hyperkaliämie und Hyponaträmie typische Befunde eines Hypoadrenokortizismus darstellen [8]. Physiologische Elektrolytkonzentrationen schließen einen isolierten Hypocortisolismus allerdings nicht aus. Infolge der Diarrhoe und der damit assoziierten Verluste wird eine Hypokaliämie bei chronischen Durchfallerkrankungen allerdings häufiger beobachtet. Das Vorliegen einer Hypocholesterolämie ist vereinbar mit einer intestinalen Malabsorption, wobei eine Hypocholesterolämie auch bei Hunden mit portosystemischem Shunt und Hypoadrenokortizismus beobachtet werden kann [8, 34].

$\mathrm{Zu}$ den weiterführenden Blutuntersuchungen im Rahmen der Abklärung von chronischer Diarrhoe gehören die Bestimmung von [18]:

- Cobalamin

- Folsäure

- Cortisol basal (bzw. ACTH-Stimulationstest)

- TLI (Trypsin-like Immunoreactivity)

- cPLI (canine Pankreaslipase)

Diese Untersuchungen müssen nicht zeitgleich mit den allgemeinen Blutuntersuchungen eingeleitet werden. Es lohnt sich allerdings, entsprechendes Material (Serum) zu asservieren, um Patient und Besitzer eine 2. Blutprobenentnahme zu ersparen. Viele Laboratorien bieten diese Parameter bereits in Form eines sogenannten „Durchfallprofils“ an. Cobalamin wird ausschließlich im Ileum absorbiert. Da Hunde eine deutlich geringere Cobalamin-Speicherkapazität als Menschen haben, kann ein Cobalaminmangel als Marker für eine Schädigung des unteren Dünndarms (Malabsorption) herangezogen werden. Auch die meisten Hunde mit einer exokrinen Pankreasinsuffizienz weisen aufgrund des Mangels des für die Resorption von Cobalamin notwendigen Intrinsic Factors eine Hypocobalaminämie auf [3].

Folsäure wird im oberen Dünndarm resorbiert. Eine niedrige Folsäurekonzentration kann somit auch einen zusätzlichen Hinweis auf eine Erkrankung des oberen Dünndarms liefern. Anders als in der Humanmedizin korrelieren Cobalamin- und Folsäurekonzentration nicht mit dem Ansprechen auf eine antibakterielle Therapie und können daher nicht zur Diagnose einer antibiotikaresponsiven Erkrankung herangezogen werden. Allerdings ermöglicht die Bestimmung der Cobalamin- und Folsäurekonzentration eine Aussage zur Lokalisation und Art der Durchfallerkrankung [12, 30].

Bei einer Basalcortisolkonzentration über $2 \mu \mathrm{g} / \mathrm{dl}$ kann das Vorliegen eines Hypoadrenokortizismus mit großer Wahrscheinlichkeit ausgeschlossen werden. Bei einer Konzentration unter $2 \mu \mathrm{g} /$ dl sollte ein ACTH-Stimulationstest zur 
sicheren Abklärung dieser Differenzialdiagnose durchgeführt werden [19, 25]. Die Bestimmung der TLI ist ein einfacher, hochsensitiver und hochspezifischer Test zum Nachweis einer exokrinen Pankreasinsuffizienz $[13,30]$.

\section{Sonografische Untersuchung des Abdomens}

Sofern Kot- und Blutuntersuchungen keinen besonderen Befund ergeben, schließt sich als nächster diagnostischer Schritt zur Aufarbeitung des Patienten mit chronischer Diarrhoe die sonografische Untersuchung des Abdomens an. Ziel der bildgebenden Diagnostik ist in erster Linie, strukturelle Veränderungen im Darm-Trakt zu lokalisieren, um über die geeignete Art der Probenentnahme entscheiden zu können. Eine Diagnosestellung ist anhand der sonografischen Untersuchung alleine jedoch nicht möglich. Trotzdem liefert der Ultraschall vor allem bei gastrointestinalen neoplastischen Erkrankungen wichtige Informationen [22]. Sofern Veränderungen der Muskularis festgestellt werden, sind die Hauptdifferenzialdiagnosen eine intestinale Neoplasie oder eine IBD (Inflammatory Bowel Disease). In diesem Fall ist häufig eine frühzeitige Biopsieentnahme indiziert, um das Vorliegen einer neoplastischen Erkrankung abklären zu können. Neben dem Gastrointestinaltrakt sollte besonderes Augenmerk auch auf die abdominalen Lymphknoten gerichtet werden ( $\triangleright$ Abb.2). Ein sonografischer Normalbefund schließt jedoch das Vorliegen einer intestinalen Neoplasie oder chronischen Entzündung nicht aus [10].

\section{Eliminationsdiät}

Sofern anhand der bislang erfolgten diagnostischen Schritte keine besonderen Befunde erhoben werden können, kann vor weiteren invasiven diagnostischen Maßnahmen eine Eliminationsdiät erfolgen. Hierbei handelt es sich gleichzeitig um eine diagnostische und therapeutische Maßnahme. Selbst ein Großteil der in Überweisungszentren vorgestellten Patienten weist trotz intensiv erfolgter Diagnostik eine futtermittelresponsive Diarrhoe (FRD) auf [4]. Eine gezielte Eliminationsdiät ist die einzige Möglichkeit, eine FRD mit Sicherheit abzuklären. Serologische Allergietests zei- gen eine schlechte Sensitivität und können die Durchführung einer Eliminationsdiät daher nicht ersetzen $[9,16]$. Kommerzielle hydrolysierte Diäten (z.B. Royal Canin Canine hypoallergenic, Hill's z/d ultra) bieten eine einfache und ausgewogene Möglichkeit zur Durchführung einer Eliminationsdiät. Das allergenarme Prinzip dieser Diäten beruht darauf, dass die enthaltenen Proteine aufgespalten sind und in Form kleinerer Peptide bzw. Aminosäuren eine geringere allergene Wirkung haben. Mit diesen Diäten können allergische Reaktionen vom Typ I ausgeschlossen werden [23]. Da Typ-IV-Reaktionen dennoch möglich sind, bietet die Fütterung einer ausgewählten Proteinquelle eine sehr gute Option [12]. Diäten, die auf einer ausgewählten Proteinquelle beruhen, stehen kommerziell zur Verfügung, können aber auch selbst zubereitet werden. In diesem Fall sollte auf eine exotische Proteinquelle zurückgegriffen werden, mit der der Patient möglichst zuvor noch nicht in Kontakt gekommen ist (z.B. Pferdefleisch, Straußenfleisch). Diese Proteinquelle wird mit einer Kohlenhydratquelle (z.B. Erbsenflocken, Kartoffel) im Verhältnis 2:1 kombiniert.

Eine gute Besitzercompliance ist bei der Ausschlussdiät unbedingt erforderlich. Daher sollte im Vorfeld mit den Besitzern besprochen werden, welche Art der Diät infrage kommt. Die Ausschlussdiät muss über mindestens 8 Wochen verabreicht werden, um eine FRD sicher ausschließen zu können. In dieser Zeit muss auf die Fütterung anderer Futtermittel außer der Diät strikt verzichtet werden. Die Besitzer sollten zudem ermuntert werden,

ein Futtermitteltagebuch zu führen, in das Durchfall, Erbrechen sowie Abweichungen von der Diät eingetragen werden, um später Rückschlüsse auf mögliche Ursachen ziehen zu können.

Wenn innerhalb der ersten 2 Wochen der Eliminationsdiät keine Besserung der Diarrhoe zu verzeichnen ist, erscheint eine FRD unwahrscheinlich.

In diesem Fall sollten weiterführende diagnostische Maßnahmen vorgezogen werden, dennoch empfiehlt es sich, die Diät beizubehalten. Die Therapie bei Erkrankungen wie der IBD basiert häufig auf einer Kombination aus hypoallergener Diät und medikamenteller antiinflammatorischer Therapie.

\section{Biopsieentnahme}

Die Biopsieentnahme stellt den letzten Schritt der diagnostischen Aufarbeitung dar, weil es sich hierbei um ein invasives Verfahren handelt, für das eine Allgemeinanästhesie notwendig ist. Sofern das Fließschema der diagnostischen Aufarbeitung verfolgt wurde und der Patient bislang keine klinische Besserung gezeigt hat bzw. keine anderweitige Diagnose gestellt werden konnte, stehen 2 Hauptdifferenzialdiagnosen im Raum: - IBD

- intestinale Neoplasie

Falls die zuvor erfolgten diagnostischen Schritte keine spezifische Lokalisation intestinaler Veränderungen ergeben haben, bietet sich eine endoskopisch gestützte Biopsieentnahme an. Hierfür ist die Klassifizierung zwischen Dünn- und

Abb. 2 Sonografisches Bild eines Hundes mit chronischem Dickdarmdurchfall und Rektumprolaps infolge eines multizentrischen Lymphoms mit Beteiligung des Gastrointestinaltraktes. Bei der rektalen Untersuchung waren multiple derbe Umfangsvermehrungen aufgefallen. Die Pfeilspitze zeigt auf einen echolosen, hochgradig vergrößerten abdominalen Lymphknoten.

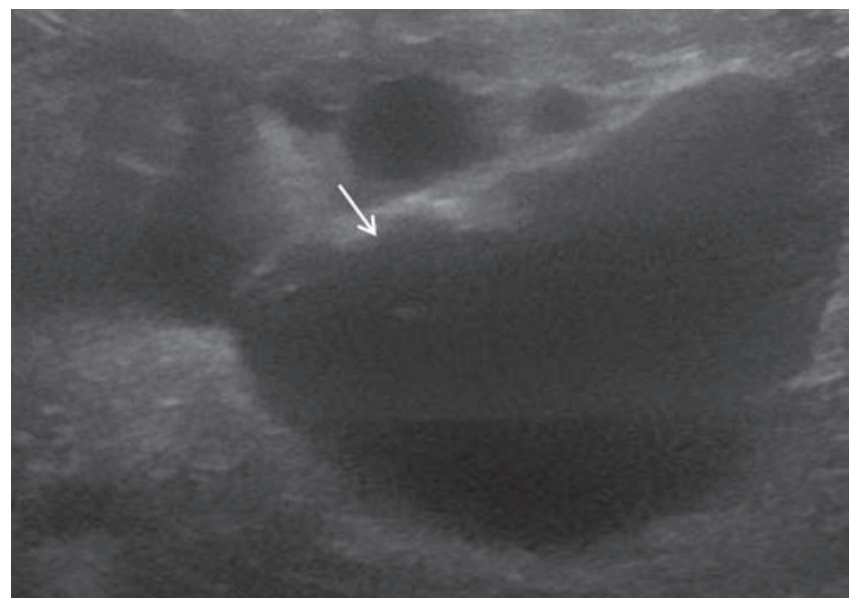




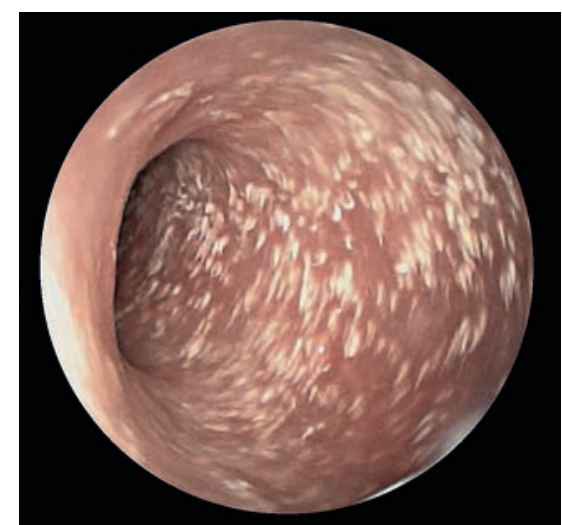

Abb. 3 Endoskopisches Bild des Duodenums eines Hundes mit Proteinverlustenteropathie. Die weißen Areale stellen hochgradige Lymphangiektasien dar.

Dickdarmdiarrhoe besonders wichtig. Bei einer reinen Dünndarmerkrankung ist eine Gastroduodenoskopie ausreichend. Bei einer reinen Dickdarmerkrankung genügt eine Koloskopie. Histologisch weisen jedoch bis zu 90\% der Hunde mit chronischem Durchfall Mischformen auf [4]. Bei Mischformen sollte die Allgemeinanästhesie für beide Verfahren genutzt werden. Mit einer Routineendoskopie kann jedoch nie der gesamte Magen-Darm-Trakt beurteilt werden (Ausnahme: Doppelballonendoskopie).

Da die pathohistologischen Befunde in verschiedenen Lokalisationen unterschiedlich sein können, wird teilweise empfohlen, in jedem Fall zusätzliche Ileumbiopsien zu entnehmen [5, 28]. Wichtig ist zudem, dass die Patienten bei Durchführung einer Koloskopie adäquat vorbereitet sein müssen. Zur Vorbereitung sollten die Patienten 1-2 Tage nüchtern sein und 1 Tag vor der geplanten
Koloskopie stationär aufgenommen werden, da Laxanzien (in der Regel über eine nasoösophageale Sonde) verabreicht werden müssen. Die Endoskopie ermöglicht eine direkte Visualisierung eventueller mukosaler Veränderungen ( $\triangleright$ Abb.3). Es wird empfohlen, mindestens 6 -8 gute Biopsien pro Lokalisation (Magen, Duodenum, Ileum, Kolon) aus unterschiedlichen Arealen zu entnehmen $[33,35]$. Sofern die Muskularis betroffen ist, ist eine endoskopisch gestützte Biopsieentnahme nicht indiziert, da hierbei nur die Mukosa und teils die Submukosa erreicht werden kann. In diesem Fall sind chirurgisch entnommene Keilbiopsien durch alle Schichten besser, wenn auch risikoreicher. Nahtdehiszenzen treten vor allem innerhalb von 3-5 Tagen nach Biopsieentnahme auf. Zudem kann durch Hypalbuminämie die Gefahr von Nahtdehiszenzen und allgemeinen Wundheilungsstörungen erhöht sein, weshalb bei Patienten mit deutlicher Hypalbuminämie auf Vollschichtbiopsien möglichst verzichtet werden sollte. Auch bei Patienten mit primärer Dickdarmdiarrhoe ist die Entnahme von Vollschicht-Biopsien aus dem Kolon aufgrund der schlechten Heilungstendenz sehr kritisch anzusehen. Entscheidend ist zudem, dass die Biopsieentnahme unbedingt vor dem Einsatz von Kortikosteroiden erfolgen muss, da eine adäquate Diagnosestellung sonst nicht möglich ist $(\triangleright$ Tab.3).

Die anschließende pathohistologische Untersuchung ermöglicht eine Aussage über die intestinale Architektur (Krypten-, Zottenaufbau), die Art und den Schweregrad der Entzündung (z.B. lymphoplasmazellulär, eosinophil, granulo- matös) sowie das Vorhandensein neoplastischer Zellen. Auch für den Pathologen ist eine Unterscheidung zwischen entzündlich und neoplastisch teilweise schwierig, weshalb im Anschluss an die pathohistologische Untersuchung oft immunhistologische Verfahren notwendig sind.

\section{Therapie und Management ausgewählter Erkrankungen}

Die Therapie richtet sich in erster Linie nach der zugrunde liegenden Ursache, kann allerdings durch unterstützende Maßnahmen ergänzt werden.

\section{Hypoadrenokortizismus}

Chronische Durchfallpatienten, bei denen ein Hypoadrenokortizismus diagnostiziert wurde, müssen nicht immer klassische Elektrolytverschiebungen (Hyponaträmie, Hyperkaliämie) aufweisen. Gerade Patienten, die chronischen Durchfall zeigen, leiden häufig an einem isolierten Glukokortikoidmangel und nicht an einem deutlichen Mineralokortikoidmangel. Ohne klassische Elektrolytverschiebungen ist eine Therapie mit Glukokortikoiden ausreichend. Lediglich die physiologische endogene Glukokortikoiddosis muss substituiert werden (Prednisolon 0,1-0,2 mg/kg alle 12-24 h). Bei zusätzlich bestehendem Mineralokortikoidmangel ist zudem der Einsatz von Fludrokortison $(0,01-0,02 \mathrm{mg} / \mathrm{kg} / \mathrm{d})$ indiziert. Die Substitution muss lebenslang erfolgen. Elektrolyte sowie Harnstoff als Parameter der Dehydratation sollten bei diesen Patienten regelmäßig kontrolliert werden, um eine Dosisanpassung vornehmen zu können [8].

Tab. 3 Vergleich zwischen endoskopischer und chirurgischer Biopsieentnahme.

\begin{tabular}{|l|l|}
\hline endoskopische Biopsieentnahme & chirurgische Biopsieentnahme (Vollschichtbiopsien) \\
\hline kostengünstiger & kostenintensiver \\
\hline risikoarm, schnell & invasiv, Gefahr der Nahtdehiszenz mit septischer Peritonitis \\
\hline nur Mukosabiopsien, lleumbiopsien teilweise schwierig & Beurteilung aller Schichten möglich, lleumbiopsien einfach möglich \\
\hline ggf. Gastroduodenoskopie und Koloskopie erforderlich & chirurgisches Verfahren erforderlich \\
\hline direkte Visualisierung veränderter Areale & Visualisierung lediglich der Serosa \\
\hline Entnahme zahlreicher Proben möglich & Biopsie anderer Organe möglich \\
\hline Diagnose von Ulzerationen, Erosionen, Lymphangiektasien & Visualisierung lediglich der Serosa
\end{tabular}


Tab. 4 Cobalaminsubstitution (@) Gastrointestinal laboratory, Texas A\&M University).

\begin{tabular}{|l|l|}
\hline Körpergewicht & Dosis \\
\hline$<5 \mathrm{~kg}$ & $250 \mu \mathrm{g}$ \\
\hline $5-15 \mathrm{~kg}$ & $400 \mu \mathrm{g}$ \\
\hline $15-30 \mathrm{~kg}$ & $800 \mu \mathrm{g}$ \\
\hline $30-45 \mathrm{~kg}$ & $1200 \mu \mathrm{g}$ \\
\hline$>45 \mathrm{~kg}$ & $1500 \mu \mathrm{g}$ \\
\hline
\end{tabular}

\section{Exokrine Pankreasinsuffizienz}

Hunde mit einer exokrinen Pankreasinsuffizienz benötigen eine Substitution der exokrinen Pankreasenzyme. Diese Substitution muss ebenfalls lebenslang erfolgen. Exokrine Pankreasenzyme stehen kommerziell in Pulverform zur Verfügung, können aber bei ungenügendem Ansprechen auf die Therapie auch in Form von Rinderpankreas oder humanmedizinischen Kapseln gegeben werden. Die Enzyme sind mit dem Futter zu verabreichen. Die Dosis richtet sich nach den klinischen Symptomen und sollte so lange hochtitriert werden, bis Gewichtszunahme und geformter Kotabsatz verzeichnet werden. Nicht selten weisen diese Patienten aufgrund des fehlenden Intrinsic Factors auch eine Hypocobalaminämie auf, die substituiert werden muss ( $\bullet$ Tab.4). Die subkutanen Injektionen von Cobalamin erfolgen laut folgendem Schema:

- $1 \times$ wöchentlich über 6 Wochen

- $1 \times$ alle 2 Wochen über 12 Wochen

- anschließend $1 \times$ monatlich

Es sollte ein reines Cobalaminprodukt gebraucht werden, da Mischprodukte von B-Vitaminen unangenehmer bei der Injektion sind und nicht genügend Cobalamin im Verhältnis zum Volumen enthalten.

\section{Futtermittelresponsive Diarrhoe (FRD)}

Futtermittelresponsive Erkrankungen lassen sich lediglich durch ein striktes Einhalten der jeweiligen Diät diagnostizieren und therapieren. Die Therapie von FRD hat den großen Vorteil, dass eine Futterumstellung hierbei genügt und kein Einsatz von zusätzlichen Medikamenten erforderlich ist, sofern die Diät strikt eingehalten wird. Wichtig ist aufgrund der lebenslangen Therapie, dass die Diät eine gute Akzeptanz hat.

\section{Inflammatory Bowel Disease (IBD)}

Anders als bei den zuvor genannten Erkrankungen ist die Ursache der Entzündung im Fall der IBD nicht bekannt. Infolge der unklaren Ätiologie gibt es keine einheitliche Therapieempfehlung für den IBD-Patienten. Grundsätzlich beruht die Therapie auf einer Kombination aus:

- Diät (zumeist hydrolisiert oder exotische, neue Proteinquelle)

- antiinflammatorischen Arzneimitteln

Aufgrund der schnellen Wirksamkeit und der geringen Medikamentenkosten werden Glukokortikoide (vor allem Prednisolon) meist als 1. Wahl eingesetzt. Angesichts des immunmediierten Charakters der Erkrankung werden initial meist immunsuppressive Dosen favorisiert (1-2 mg/kg alle $12 \mathrm{~h}$ ). Allerdings existiert keine einheitliche Meinung bezüglich der zu verwendenden Dosis. Auch lokal enteral wirkende Arzneimittel, wie z.B. Budenosid, haben Einzug in die Veterinärmedizin gehalten. Die erhoffte Reduktion systemischer Nebenwirkungen durch lokal wirkende Arzneimittel ist allerdings weniger gut als erhofft, da auch bei Verwendung dieser

Tab. 5 Therapie der IBD beim Hund.

\begin{tabular}{|c|c|c|}
\hline Therapie & wirksamer Bestandteil & Dosierung \\
\hline Diät & $\begin{array}{l}\text { neue Proteinquelle } \\
\text { hydrolysierte Diät } \\
\text { (jeweils über mindes- } \\
\text { tens 6-8 Wochen) }\end{array}$ & - \\
\hline \multirow[t]{5}{*}{$\begin{array}{l}\text { immunsuppressi- } \\
\text { ve Therapie }\end{array}$} & Prednisolon & $\begin{array}{l}1-2 \mathrm{mg} / \mathrm{kg} \text { alle } 12 \mathrm{~h} \text { p.o. } \\
\text { (Dosisreduktion nach Verschwinden } \\
\text { der klinischen Symptome) }\end{array}$ \\
\hline & Budesonid & $1-3 \mathrm{mg} / \mathrm{kg}$ alle $24 \mathrm{~h}$ p.o. \\
\hline & Cyclosporin & 5 mg/kg alle 24 h p.o. \\
\hline & Chlorambucil & $\begin{array}{l}4-6 \mathrm{mg} / \mathrm{m}^{2} \text { alle } 24 \mathrm{~h} \text { p.o. } \\
\text { (über } 7-21 \text { Tage, dann Dosisreduktion) }\end{array}$ \\
\hline & Azathioprin & $1-2 \mathrm{mg} / \mathrm{kg}$ alle 24 h p.o. \\
\hline \multirow{3}{*}{$\begin{array}{l}\text { antimikrobielle } \\
\text { Therapie }\end{array}$} & Metronidazol & $10-15 \mathrm{mg} / \mathrm{kg}$ alle $12 \mathrm{~h}$ p.o. \\
\hline & Tylosin & $\begin{array}{l}10-40 \mathrm{mg} / \mathrm{kg} \text { alle } 12 \mathrm{~h} \text { p.o. } \\
25 \mathrm{mg} / \mathrm{kg} \text { alle } 24 \text { h p.o. }\end{array}$ \\
\hline & Enrofloxacin & $\begin{array}{l}\text { 5-10 mg/kg alle } 24 \text { h p.o. } \\
\text { (histiozytäre ulzerative Kolitis) }\end{array}$ \\
\hline
\end{tabular}

Arzneimittel eine deutliche Suppression der Hypophysen-NebennierenrindenAchse nachgewiesen werden konnte [31]. Nicht alle IBD-Patienten sprechen auf Prednisolon an. Zudem ist langfristig eine hohe Dosis aufgrund der systemischen Nebenwirkungen zu vermeiden. Sollte die Dosis nicht reduziert werden können oder das Ansprechen auf die Therapie ungenügend sein, ist eine Kombination mit anderen antiinflammatorischen Arzneimitteln indiziert. Leider gibt es auch diesbezüglich keine einheitliche Empfehlung. Möglich ist unter anderem der Einsatz von:

- Cyclosporin A (5 mg/kg alle 24 h p.o.)

- Azathioprin (1-2 mg/kg alle 24-48 h p.o.)

- Chlorambucil (2-6 mg/m² alle 24 h p.o.)

Jedes dieser Arzneimittel hat seine Vorund Nachteile. Cyclosporin ist vor allem sehr kostenintensiv und kann selbst Durchfall auslösen. Azathioprin ist kos-

\section{konkret}

Ziel der IBD-Therapie ist eine Kombination aus allergenarmer Diät und antiinflammatorischen Arzneimitteln zu finden, unter der der Patient stabil ist und keine Nebenwirkungen zeigt. 
tengünstig, kann aber mit schwerwiegenden Nebenwirkungen assoziiert sein (Myelosuppression, Hepatotoxizität, Pankreatitis).

Sobald dieser stabile Zustand erreicht ist, sollte die Medikamentendosis reduziert werden. In den besten Fällen können die Arzneimittel ganz abgesetzt werden, und der Patient kann langfristig nur mit der Diät stabil gehalten werden. In den übrigen Fällen ist das Ziel eine möglichst niedrige Dosis zu erreichen, unter der keine klinischen Symptome auftreten. Die wichtigsten therapeutischen Möglichkeiten sind in $>$ Tab. 5 zusammengefasst.

Bei primären Dickdarmerkrankungen zeigen zudem der Einsatz von löslichen Fasern (Psyllium) und/oder Metronidazol (10-15 mg/kg alle 12 h p.o. für 3-7 Tage) einen guten Effekt. Eine Ausnahme stellt die histiozytäre ulzerative Kolitis des Boxers dar, bei der als Grundursache ein spezifischer enteroinvasiver E. coliStamm nachgewiesen werden konnte. Dieser kann nur mit Fluoreszenz-in-situHybridisierung (FISH) oder Kultivierung von Biopsien nachgewiesen werden, jedoch nicht mit einer klassischen bakteriellen Kultur. Die betroffenen Hunde konnten erfolgreich mit Enrofloxacin ( $5 \mathrm{mg} / \mathrm{kg} / 24 \mathrm{~h}$ p.o.) über 4-8 Wochen therapiert werden [14].

\section{Proteinverlustenteropathie (PVE)}

Die PVE stellt eine Sonderform der Durchfallerkrankungen dar. Sie kann als Folge anderer Darmerkrankungen auftreten (z.B. IBD, intestinale Neoplasie), mit schwerwiegenden Lymphangiektasien assoziiert sein (z.B. bei Yorkshire Terrier und Rottweiler) oder rassespezifisch vorkommen (Basenji-Enteropathie, Enteropathie des Irish Soft Coated Wheaten Terrier). Bei Patienten mit einer Hypoproteinämie ist eine zügige diagnostische Aufarbeitung wichtig, da infolge der Hypoproteinämie das Thrombembolierisiko hoch ist und die Hypoproteinämie mit einem schlechten Überleben assoziiert ist. Essenziell ist vor allem der Ausschluss möglicher Differenzialdiagnosen einer Hypalbuminämie (Proteinverlustnephropathie, Hepatopathie). Empfohlen werden bei diesen Patienten Diäten, die fettreduziert sind $(<15 \%)$ und hochverdauliche Proteine enthalten, z.B.
Royal Canin Canine gastrointestinal low fat [26]. Wichtig ist zudem die Grundursache zu diagnostizieren und entsprechend zu behandeln, um einen weiteren Proteinverlust zu reduzieren. Häufig beinhaltet die Therapie daher eine antiinflammatorische Medikation (z.B. Prednisolon $1-2 \mathrm{mg} / \mathrm{kg}$ alle $12-24 \mathrm{~h}$ p.o.) und eine medikamentelle Reduktion des Thrombembolierisikos (z.B. Clopidogrel $1-2 \mathrm{mg} / \mathrm{kg}$ alle $24 \mathrm{~h}$ p.o.).

\section{Intestinale Neoplasien}

$\mathrm{Zu}$ den häufigsten intestinalen Neoplasien des Hundes gehören:

- Lymphom

- Adenokarzinom

- gastrointestinale stromale Tumoren

Die Therapie der Wahl bei neoplastischen Erkrankungen des Gastrointestinaltraktes ist die chirurgische Entfernung. Gerade bei Patienten mit chronischer Diarrhoe als Leitsymptom handelt es sich bei neoplastischen Erkrankungen meist um diffus-infiltrativ wachsende Neoplasien. Bei diesen Tumoren ist die Therapie der Wahl eine Chemotherapie. Für lymphatische Erkrankungen existieren entsprechende Chemotherapieprotokolle. Bei gastrointestinalen stromalen Tumoren kann der Einsatz von Tyrosinkinaseinhibitoren (z.B. Masitinib) einen positiven Effekt haben [12].

\section{Zusätzliche Maßnahmen}

Unkompliziert und in der Regel ohne Nebenwirkungen ist der Einsatz sogenannter Styptika (z.B. Enterogelan ${ }^{\circledR}$, Möhrchenpellets), die eine koteindickende Wirkung haben und somit Durchfall reduzieren sollen. Kritisch diskutiert werden sollte der Einsatz solch kommerzieller Produkte allerdings in Kombination mit einer Eliminationsdiät, da enthaltene Kohlenhydrate und Proteine eine allergene Wirkung haben können. Wichtig ist zudem, dass durch den Einsatz eines Styptikums die Kotkonsistenz verändert wird und somit nicht mehr als Parameter zur Beurteilung des Therapieerfolgs herangezogen werden kann.

Gerade bei IBD-Patienten können unterstützend auch ungesättigte Fettsäuren infolge des antiinflammatorischen Effekts eingesetzt werden [27]. Bislang feh- len Daten, die einen positiven Effekt des Einsatzes von Probiotika und Präbiotika bei Hunden mit chronischer Diarrhoe belegen. Von Vorteil ist, dass diese Präparate in der Regel wenig Nebenwirkungen haben und daher versuchsweise eingesetzt werden können.

\section{Prognose}

Die Prognose bei Patienten mit chronischer Diarrhoe hängt in erster Linie von der Ursache ab. Endoparasitosen, Pankreasinsuffizienz und Hypoadrenokortizismus sind Erkrankungen, die bei adäquater Therapie eine gute Prognose haben, auch wenn die Therapie bei den zuletzt genannten beiden Erkrankungen lebenslang erforderlich ist. Auch futtermittelresponsive Erkrankungen sind gut zu therapieren. Allerdings kann es hierbei zwischenzeitlich immer wieder zu Rückschlägen kommen, vor allem wenn die Einhaltung der Diät Probleme bereitet. Bei Patienten mit IBD ist die Prognose sehr variabel und hängt vor allem vom Ansprechen auf die jeweilige Therapie und dem Albuminwert ab [7]. Intestinale Neoplasien sind meist mit einer schlechten Prognose verbunden. Eine Heilung ist in diesen Fällen in der Regel nicht möglich. Ziel ist somit lediglich die Remission der Erkrankung.

Als prognostisch schlechte Faktoren bei Hunden mit chronischer Enteropathie konnten unter anderem eine Hypalbuminämie sowie Hypocobalaminämie identifiziert werden [2,7]. Zudem scheinen bestimmte Erkrankungen wie die PLE bei einzelnen Hunderassen (Rottweiler) mit einem besonders schlechten Überleben assoziiert zu sein [6].

\section{Literatur}

1 Allenspach K. Diseases of the large intestine. In: Ettinger SJ, Feldmann EC, eds. Textbook of Veterinary Internal Medicine. $7^{\text {th }}$ ed. St. Louis Missouri: Saunders Elsevier; 2010: 1573-1594

2 Allenspach K, Wieland B, Gröne A, Gaschen F. Chronic enteropathies in dogs: evaluation of risk factors for negative outcome. J Vet Intern Med 2007; 21: 700-708

3 Batchelor DJ, Noble PJ, Taylor RH et al. Prognostic factors in canine exocrine pancreatic insufficiency: prolonged survival is likely if clinical remission is achieved. J Vet Intern Med 2007; 21: 54 
4 Burgener IA, König A, Allenspach K et al. Upregulation of Toll-like receptors in chronic enteropathies in dogs. J Vet Intern Med 2008; 22: $553-560$

5 Casamian-Sorrosal D, Willard MD, Murray JK et al. Comparison of histopathologic findings in biopsies from the duodenum and ileum of dogs with enteropathy. J Vet Intern Med 2010; 24: 80-83

6 Dijkstra M, Kraus JS, Bosje JT, Den Hertog E. Protein-losing enteropathy in Rottweilers. Tijdschr Diergeneeskd 2010; 135: 406-412

7 Equilino M, Théodoloz V, Gorgas D et al. Evaluation of serum biochemical marker concentrations and survival time in dogs with protein-losing enteropathy. J Am Vet Med Assoc 2015; 246: 91-99

8 Feldmann EC, Nelson RW. Canine and feline endocrinology and reproduction. $3^{\text {rd }}$ ed. St. Louis, Missouri: Saunders; 2004: 394-439

9 Foster AP, Knowles TG, Moore AH et al. Serum IgE and IgG responses to food antigens in normal and atopic dogs, and dogs with gastrointestinal disease. Vet Immunolog Immunopathol 2003; 92: 113-124

10 Frances M, Lane AE, Lenard ZM. Sonographic features of gastrointestinal lymphoma in 15 dogs. J Small Anim Prac 2013; 54: 468-474

11 German AJ, Hall EJ, Day MJ. Chronic intestinal inflammation and intestinal disease in dogs. J Vet Intern Med 2003; 17: 8-20

12 Hall EJ, German AJ. Diseases of the small intestine. In: Ettinger SJ, Feldmann EC, eds. Textbook of Veterinary Internal Medicine. $7^{\text {th }}$ ed. St. Louis, Missouri: Saunders Elsevier; 2010: 1526-1572

13 Heilmann RM, Steiner JM. Current Veterinary Therapy: Laboratory Testing for the Exocrine Pancreas. In: Bonagura JD, Twedt DC, eds. Kirk's Current Veterinary Therapy XV. St. Louis, Missouri: Saunders Elsevier; 2014 : 554-557

14 Hostutler RA, Luria B], Johnson SE et al. Antibiotic-responsive histiocytic ulcerative colitis in 9 dogs. J Vet Intern Med 2004; 18 : 499-504

15 Janeczko S, Atwater D, Bogel E et al. The relationship of mucosal bacteria to duodenal histopathology, cytokine mRNA, and clinical disease activity in cats with inflammatory bowel disease. Vet Microbiol 2008; 128: 178

16 Jeffers JG, Shanley KJ, Meyer EK. Diagnostic testing of dogs for food hypersensitivity. J Am Vet Med Assoc 1991; 198: 245-250
17 Jergens AE. Current Veterinary Therapy: Antibiotic Responsive Enteropathy. In: Bonagura JD, Twedt DC, eds. Kirk's Current Veterinary Therapy XV. St. Louis, Missouri: Saunders Elsevier; 2014: 518-522

18 Kathrani A, Steiner JM, Suchodolski j et al. Elevated canine pancreatic lipase immunoreactivity concentration in dogs with inflammatory bowel disease is associated with a negative outcome. J Small Anim Prac 2009; 50: 126-132

19 Kintzer PP, Peterson MA, Kintzer PP. Primary and secondary hypoadrenocorticism. Vet Clin North Am Small Anim Pract 1997; 27: 349-357

20 Klein SC, Peterson ME. Canine hypoadrenocorticism: Part I. Can Vet J 2010; 51: 63-69

21 Leib MS. Treatment of chronic idiopathic large-bowel diarrhea in dogs with a highly digestible diet and soluble fiber: a retrospective review of 37 cases. J Vet Intern Med 2000; 14: 27-32

22 Leib MS, Larson MM, Grant DC et al. Diagnostic utility of abdominal ultrasonography in dogs with chronic diarrhea. J Vet Intern Med 2012; 26: 1288-1294

23 Mandigers PJJ, Biourge V, Van den Ingh TSGAM et al. A randomized, open-label, positively-controlled field trial of a hydrolyzed diet in dogs with chronic small bowel enteropathy. J Vet Intern Med 2010; 24: 1350-1357

24 Marchetti V, Lubas G, Lombardo A et al. Evaluation of erythrocytes, platelets, and serum iron profile in dogs with chronic enteropathy. Vet Med Int 2010; doi: $10.4061 / 2010 / 716040$

25 Nelson RW. Hypoadrenocortizismus. In: Nelson RW, Couto CG, eds. Innere Medizin der Kleintiere. München: Urban und Fischer; 2006: 860-865

26 Okanishi H, Yoshioka R, Kagawa Y, Watari T. The clinical efficacy of dietary fat restriction in treatment of dogs with intestinal lymphangiectasia. J Vet Intern Med 2014; 28: 809-817

27 Ontsouka CE, Burgener IA, Mani O, Albrecht C. Polyunsaturated fatty acid-enriched diets used for treatment of canine chronic enteropathies decrease the abundance of selected genes of cholesterol homeostasis. Domest Anim Endocrin 2010; 38: 32-37

28 Procoli F, Mõtsküla PF, Keyte SV et al. Comparison of histopathologic findings in duodenal and ileal endoscopic biopsies in dogs with chronic small intestinal enteropathies. J Vet Intern Med 2013; 27: 268-274
29 Simpson K, Dogan B, Rishniw M et al. Adherent and invasive Escherichia coli is associated with granulomatous colitis in boxer dogs. Infect Immun 2006; 74: 4778

30 Steiner JM. Review of commonly used clinical pathology parameters for general gastrointestinal disease with emphasis on small animals. Toxicol Pathol 2014; 42: 189-194

31 Stroup ST, Behrend EN, Kemppainen RJ, Smith-Carr S. Effects of oral administration of controlled-ileal-release budesonide and assessment of pituitary-adrenocortical axis suppression in clinically normal dogs. Am J Vet Res 2006; 67: 1173-1178

32 Suchodolski JS, Xenoulis PG, Paddock CG et al. Molecular analysis of the bacterial microbiota in duodenal biopsies from dogs with idiopathic inflammatory bowel disease. Vet Microbiol 2010; 142: 394

33 Washabau RJ, Day MJ, Willard MD et al. Endoscopic, biopsy, and histopathologic guidelines for the evaluation of gastrointestinal inflammation in companion animals. J Vet Intern Med 2010; 24: 10-26

34 Willard MD. Diarrhea. In: Ettinger SJ, Feldmann EC, eds. Textbook of Veterinary Internal Medicine. $7^{\text {th }}$ ed. St. Louis, Missouri: Saunders Elsevier; 2010: 201-206

35 Willard MD, Moore GE, Denton BD et al. Effect of tissue processing on assessment of endoscopic intestinal biopsies in dogs and cats. J Vet Intern Med 2010; 24: 84-89

\section{Dr. Dorothee Dahlem}

Klinik für Kleintiere

Justus-Liebig-Universität Gießen

Frankfurter Straße 126

\section{Gießen}

\section{Prof. Dr. Iwan A. Burgener}

Dipl. ACVIM \& ECVIM-CA, FTA Innere Medizin

der Klein- und Heimtiere

Chair of Internal Medicine of Companion Animals Universiteitskliniek voor Gezelschapsdieren

Yalelaan 108

3584 CM Utrecht (NL) 


\section{Fragebogen}

\section{Frage 1}

Welche der folgenden Ursachen von Diarrhoe zählt zu den intestinalen Ursachen?
a. Hepatopathien
b. Pankreatitis
c. Salmonellose
d. Staupe
e. Hypoadrenokortizismus

\section{Frage 2}

Welches der folgenden Merkmale ist hinweisend auf das Vorliegen von Dickdarmdurchfall?
a. Vomitus
b. Gewichtsverlust
c. Hämatochezie
d. Meläna
e. großes Kotvolumen

\section{Frage 3}

Welche der im Folgenden genannten Differenzialdiagnosen der chronischen Diarrhoe des Hundes zählt zu den extraintestinalen Ursachen von Diarrhoe?

a. exokrine Pankreasinsuffizienz

b. Futtermittelunverträglichkeit

c. Endoparasitose

d. Inflammatory Bowel Disease

e. intestinales Lymphom

\section{Frage 4}

Welches ist der 1. Schritt zur diagnostischen Aufarbeitung eines Hundes mit chronischer Diarrhoe und gutem Allgemeinbefinden?

a. Blutuntersuchung

b. sonografische Untersuchung des Abdomens c. radiologische Untersuchung des Abdomens

d. parasitologische Kotuntersuchung e. endoskopische Untersuchung

\section{Frage 5}

Welche der folgenden Erkrankungen sollte bei Nachweis einer Eosinophilie bei einem Hund mit chronischer Diarrhoe als Differenzialdiagnose in Betracht gezogen werden?

a. lymphoplasmazelluläre IBD

b. exokrine Pankreasinsuffizienz

c. Azotämie

d. Hepatopathie

e. Hypoadrenokortizismus

\section{Frage 6}

Welcher der folgenden blutchemischen Befunde weist auf die möglichen Differenzialdiagnosen Malabsorption, Hypoadrenokortizismus und portosystemischer Shunt hin?
a. Hypokaliämie
b. Hyponaträmie
c. Hypocholesterolämie
d. Hyperkaliämie
e. Hypochloridämie

\section{Frage 7}

Welches klinische Symptom eines Hundes mit chronischer Diarrhoe ist hinweisend auf eine Dickdarmbeteiligung?
a. Borborygmus
b. Tenesmus
c. Salivation
d. Flatulenz
e. Gewichtsverlust

\section{Frage 8}

In welchem Teil des Darm-Traktes wird Cobalamin resorbiert?
a. Duodenum
b. Jejunum
c. Ileum
d. Zäkum
e. Kolon

\section{Frage 9}

Welche Aussage in Bezug auf endoskopisch gestuitzte Biopsieentnahmen aus dem Gastrointestinaltrakt ist falsch? Die endoskopisch gestützte Biopsieentnahme...

a. ...erreicht die Tunica muscularis.

b. ...ermöglicht die Entnahme zahlreicher Biopsien.

c. ...ist einfach und schnell durchführbar.

d. ...ermöglicht die Visualisierung veränderter Schleimhautareale. e. ...ist risikoarm und kostengünstig.

\section{Frage 10}

Welches Arzneimittel gilt als Therapie der Wahl bei der histiozytären ulzerativen Kolitis des Boxers?
a. Tylosin
b. Prednisolon
c. Azathioprin
d. Enrofloxacin
e. Chlorambucil 


\section{Chronische Diarrhoe beim Hund}

Dorothee Dahlem, Iwan Burgener

A Lernerfolgskontrolle

\section{Bitte kreuzen Sie die richtigen Antworten an! Es ist jeweils nur 1 Antwort pro Frage richtig!}

\section{B Teilnehmer}

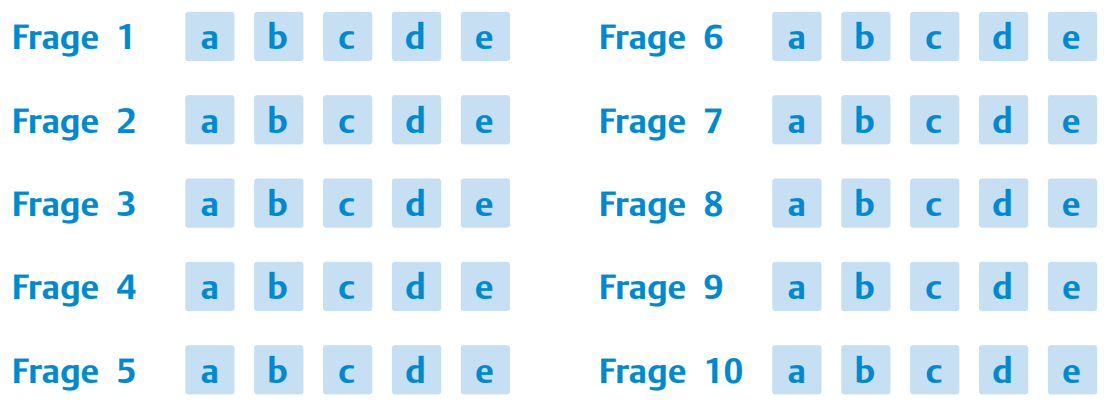

\section{Ihr Ergebnis wird vom Verlag ausgefüllt}

\section{E Erklärung}

Ich versichere, dass ich die Beantwortung der

Fragen selbst und ohne Hilfe durchgeführt habe.

Sie haben von $\quad \square$ bestanden und 1 ATF-Stunde erhalten.

Stuttgart, den

D Teilnahmebedingungen für Abonnenten der kleintier konkret kostenlos

Für diese Fortbildungseinheit können Sie 1 ATFFortbildungsstunde anerkannt bekommen. Hierfür

- müssen mindestens 70 \% der Fragen richtig beantwortet sein.

- muss der Antwortbogen vollständig ausgefüllt sein. Unvollständig ausgefüllte Bögen können nicht berücksichtigt werden!
- muss im markierten Feld* Ihre Abonnentennummer eingetragen oder eine kleintier konkret-CVE-Wertmarke aufgeklebt sein.

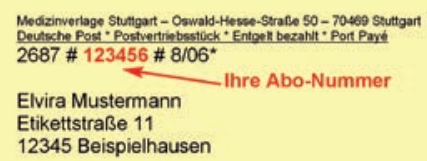

kleintier konkret-CVE-Wertmarken für NichtAbonnenten können beim Verlag zu folgenden Bedingungen erworben werden: 6erPackWertmarken, Preis 49,95€ inkl. MWSt., Artikel-Nr. 903000.

Bitte richten Sie die Bestellungen an: MVS Medizinverlage Stuttgart,

KundenServiceCenter Buch, Postfach 3011 20, 70451 Stuttgart.

${ }^{*}$ Nicht-Abonnenten bitte hier kleintier konkret-CVE-Wertmarke aufkleben, Abonnenten bitte Abonnentennummer eintragen 